\title{
OC-068 ENDOTHERAPY OF HIGH GRADE DYSPLASIA AND EARLY CANCER IN BARRETT'S OESOPHAGUS: RESULTS FROM A LARGE UK SERIES COMPARING OUTCOMES OF TWO RADICALLY DIFFERENT APPROACHES
}

doi:10.1136/gut.2011.239301.68

J-EAbela, ${ }^{1,}$ P Bhandari, ${ }^{2}$ J Morris, ${ }^{3}$ P Basford, ${ }^{2}$ C K Mackay, ${ }^{1} \mathrm{~J} J$ Going, ${ }^{4}$ D MacArthur, ${ }^{1}$ D Poller, ${ }^{5}$ D Cowlishaw, ${ }^{5}$ P Glen, ${ }^{1}$ G M Fullarton ${ }^{1}$ Department of Surgery, Glasgow Royal Infirmary, Glasgow, UK; ${ }^{2}$ Department of Gastroenterology, Queen Alexandra Hospital, Portsmouth, UK; ${ }^{3}$ Department of Gastroenterology, Glasgow Royal Infirmary, Glasgow, UK; ${ }^{4}$ Department of Pathology, Glasgow Royal Infirmary, Glasgow, UK; ${ }^{5}$ Department of Pathology, Queen Alexandra Hospital, Portsmouth, UK

Introduction Endoscopic therapy of Barrett's Oesophagus (BE) with high grade dysplasia (HGD) and/or intramucosal cancer (IMC) by endoscopic mucosal resection (EMR) or radiofrequency ablation (RFA) is a recognised alternative to surgical resection. The aim of this study is to compare the outcomes of two radically different endotherapy approaches of focal EMR of neoplastic foci followed by RFA versus focal EMR followed by more radical EMR as necessary.

Methods Patients referred with Barrett's HGD and/or IMC where treated with either focal EMR to visibly elevated lesions with subsequent intensive RFA of residual BE (Group A) or by a protocol of focal EMR to eradicate neoplasia (Group B). There were 37 patients ( $83 \%$ males) in Group A with a mean age of 66 years and a mean Barrett's segment length was $6.9 \mathrm{cms}(1-14)$. In Group B we had 44 patients ( $90 \%$ males) with a mean age of 68 years and mean segment length of $5.0 \mathrm{cms}(2-15)$.

All patients were followed up in a rigorous endoscopic programme. The mean duration of follow-up was 23 months in Group A and 32 months in Group B.

Results In Group A total of 23 EMR procedures were performed with a mean of 0.6 sessions per patient. Treatment then proceeded with a RFA-intensive protocol such that a total of 79 ablations were administered with a mean of 2 sessions per patient. Thereafter, Nd:YAG laser was applied to residual islands less than $0.5 \mathrm{~cm}$ in length.

In Group B a total of 72 EMR procedures were performed with a mean of 1.6 sessions per patient. In this group RFA was employed in 6 patients only.

No significant post-procedural complications were identified in these patients. Eradication of neoplasia (HGD and IMC) was achieved in 35/37 patients (95\%) in Group A and 41/41 $(100 \%)$ in Group B. Furthermore, during this relatively short follow-up period eradication of Barrett's oesophagus was achieved in 31/37 of patients (84\%) in Group A.

Conclusion This is the largest reported series demonstrating similarly excellent outcomes for two radically different endoscopic approaches for the ablation of Barrett's neoplasia. In addition, the RFA-intensive strategy has proved effective in eradicating residual Barrett's oesophagus. These observations 
call for a randomised controlled trial comparing these two strategies in order to identify the most cost-effective option.

Competing interests None.

Keywords Barrett's oesophagus, early cancer, endoscopic

mucosal resection, high grade dysplasia (HGD), radiofrequency ablation (RFA). 